\title{
Stage IIB Mycosis Fungoides and Sezary Syndrome AJCC v7
}

National Cancer Institute

\section{Source}

National Cancer Institute. Stage IIB Mycosis Fungoides and Sezary Syndrome A/CC V7.

NCI Thesaurus. Code C88155.

Stage IIB includes: T3, N0-2, M0, B0-1. T3: One or more tumors (equal or greater than 1 cm diameter). N0: No clinically abnormal peripheral lymph nodes; biopsy not required. N1: Clinically abnormal peripheral lymph nodes; histopathology Dutch grade 1 or NCI LN0-2. N2: Clinically abnormal peripheral lymph nodes; histopathology Dutch grade 2 or $\mathrm{NCl}$ LN3. M0: No visceral organ involvement. B0: Absence of significant blood involvement: $5 \%$ or less of peripheral blood lymphocytes are atypical (Sezary cells). B1: Low blood tumor burden: more than 5\% of peripheral blood lymphocytes are atypical (Sezary cells) but does not meet the criteria of B2. (AJCC 7th ed.) 